OPEN ACCESS

Edited by:

Chao Liu,

Beijing Normal University, China

Reviewed by:

Wei Liao,

University of Electronic Science and Technology of China, China

Susanne Koot,

VU University Amsterdam,

Netherlands

*Correspondence:

Tao Suo

suotao810815@163.com

Tingyong Feng

fengty0@swu.edu.cn

Specialty section:

This article was submitted to

Decision Neuroscience,

a section of the journal

Frontiers in Neuroscience

Received: 23 October 2018

Accepted: 19 February 2019

Published: 11 March 2019

Citation:

Wang M, Chen Z, Zhang S, Xu T, Zhang R, Suo T and Feng T (2019)

High Self-Control Reduces Risk Preference: The Role of Connectivity Between Right Orbitofrontal Cortex and Right Anterior Cingulate Cortex.

Front. Neurosci. 13:194

doi: 10.3389/fnins.2019.00194

\section{High Self-Control Reduces Risk Preference: The Role of Connectivity Between Right Orbitofrontal Cortex and Right Anterior Cingulate Cortex}

\author{
Mengmeng Wang ${ }^{1,2}$, Zhiyi Chen ${ }^{3}$, Shunmin Zhang ${ }^{3}$, Ting $\mathrm{Xu}^{3}$, Rong Zhang ${ }^{3}$, Tao Suo ${ }^{1,2 *}$ \\ and Tingyong Feng ${ }^{3,4 *}$ \\ ${ }^{1}$ School of Education, Institute of Cognition, Brain, and Health, Henan University, Kaifeng, China, ${ }^{2}$ School of Education, \\ Institute of Psychology and Behavior, Henan University, Kaifeng, China, ${ }^{3}$ Faculty of Psychology, Southwest University, \\ Chongqing, China, ${ }^{4}$ Key Laboratory of Cognition and Personality, Ministry of Education, Chongqing, China
}

Risk preference, the preference for risky choices over safe alternatives, has a great impact on many fields, such as physical health, sexual safety and financial decision making. Ample behavioral research has attested that inadequate self-control can give rise to high risk preference. However, little is known about the neural substrates underlying the effect of self-control on risk preference. To address this issue, we combined voxel-based morphometry (VBM) with resting-state functional connectivity (RSFC) analyses to explore the neural basis underlying the effect of self-control on risk preference across two independent samples. In sample 1 (99 participants; 47 males; $20.37 \pm 1.63$ years), the behavioral results indicated that the scores of selfcontrol were significantly and negatively correlated with risk preference (indexed by gambling rate). The VBM analyses demonstrated that the higher risk preference was correlated with smaller gray matter volumes in right orbitofrontal cortex (rOFC) and right posterior parietal cortex. In the independent sample 2 (80 participants; 33 males; $20.33 \pm 1.83$ years), the RSFC analyses ascertained that the functional connectivity of rOFC and right anterior cingulate cortex ( $\mathrm{rACC}$ ) was positively associated with risk preference. Furthermore, the mediation analysis identified that self-control mediated the impact of functional connectivity of rOFC-rACC on risk preference. These findings suggest the functional coupling between the rOFC and rACC might account for the association between self-control and risk preference. The present study extends our understanding on the relationship between self-control and risk preference, and reveals possible neural underpinnings underlying this association.

Keywords: self-control, risk preference, voxel-based morphometry, resting-state functional connectivity, mediation

\section{INTRODUCTION}

The decisions we make in our daily lives have a pronounced effect on our physical, psychological, and individual or family economics. Specifically, risks and uncertainties pervade our decisions across the lifespan, including physical health (Lusk and Coble, 2005), sexual safety (Harbison et al., 2018), and financial decision making (Engelmann and Tamir, 2009; 
Angkinand and Wihlborg, 2010; Wen et al., 2014). Notably, risk preference, which is defined as the general tendency to take risks in a particular decision context (Sitkin and Pablo, 1992; Mullins and Forlani, 2005), is a crucial indicator of risk decision making. Individuals with high risk preferences are commonly associated with more maladaptive behaviors, including alcohol consumption, drug abuse, smoking, gambling, and unsafe sexual activity (Donohew et al., 2000; Robbins and Bryan, 2004; Bechara, 2005; Bickel et al., 2012). Prior literature has indicated that risk preference results from a lack of self-control (Freeman and Muraven, 2010; Ryan et al., 2013). Although the relationship between self-control and risk preference has been explored in the behavioral field, little is known about the neural substrates underlying the effects of self-control on risk preference.

A large number of human decisions involving a balance between anticipated rewards and risks are regulated by selfcontrol, which can resist immediate temptations in favor of long-term goals (Kool et al., 2013). The dual-system model, a more systematic explanation for risk behavior, provides a reliable explanation for the impact of self-control on risk preference. According to this theory, risk behavior is recognized as the result of competition between the instinctive affective system and the controlled deliberative system (Casey et al., 2008; Steinberg, 2008). The instinctive affective system, also known as the "hot" system, is spontaneous and automatic, relying upon affective input, such as the expectation of reward following risk behavior (Galvan et al., 2006, 2010). Notwithstanding this, the controlled deliberative system (the "cool" system) is characterized as involving more purely cognitive processes. The affective system can be easily triggered, for example by affectively pursuing rewards due to high risk preference. Meanwhile, the controlled system could block these affective impulses and facilitate deliberative decision making (Cohen, 2005; Knoch and Fehr, 2010). Self-control has been quintessentially deemed a part of the controlled deliberative system (Kruglanski, 2018). This system underlies goal-directed behavior and requires a volitional control or willpower to be effective (Metcalfe and Mischel, 1999). However, the avoidance of risk preference requires effective selfcontrol (de Ridder et al., 2012), which is initiated by inner responses and undesired behavioral tendencies (Tangney et al., 2004; Carver and Scheier, 2012). According to the process model of self-control depletion, self-control failure is caused by the motivated switching to "want-go" goals, which are carried out for personal enjoyment and gratification, such as reward seeking (Inzlicht et al., 2014). Taken together, risk behaviors are performed because the motivation to rewards cannot be resisted by low cognitive control ability. Accordingly, it is presumable that the relative strength of the reward evaluation system compared to the cognitive control system should be highlighted as a core component of the association between self-control and risk preference.

The dual system model comprises of two distinct neurobiological sub-systems: the "cognitive control" system, which mainly involves the lateral prefrontal cortex (IPFC), parietal cortices (PC), anterior cingulate cortex (ACC) (Steinberg, 2008), and the "socioemotional" system, which is located in the limbic and paralimbic areas of the brain, such as the ventral striatum and orbitofrontal cortex (OFC). Specifically, in the "cognitive control" system, the IPFC is closely linked to deliberative processing and self-control in the suppression of affective impulses (Mcclure and Bickel, 2015; Su et al., 2018). The IPFC implements control in part by biasing processing through the connection with the posterior parietal cortex (PPC). The activation of the PPC is associated with attending to and evaluating the risks involved in decision making (Huettel et al., 2005; Christopoulos et al., 2009). The lPFC, which is responsible for executive cognitive control, might also collaborate with regions implicated in conflict monitoring, such as the ACC (Han et al., 2012; Gowin et al., 2013). In the "socioemotional" system, this striatum is believed to encode the communication between expected value and received rewards in risk decisions (Breiter et al., 2001; Tobler et al., 2009; Wang et al., 2015). Neural activity in the OFC encodes possible rewards by integrating the history of the latest outcome into expected outcomes (Elliot, 1999; Ernst et al., 2002; Akitsuki et al., 2003). Meanwhile, the hyperactivity of the OFC has been observed when individuals were engaged in risk-taking behavior (Jentsch et al., 2010). In some pathological studies, the OFC has been found to be activated in obsessive compulsive disorders (Breiter et al., 1996; Mataix-Cols et al., 2004) and in cocaine abusers (Arana et al., 2003); this region is involved with processing the reward values of stimuli and motivating behavioral responding to rewards (Goldstein et al., 2007). The OFC, once activated, needs cognitive control to allow individuals to, for example, not take a drug (Volkow and Fowler, 2000), but individuals with drug addictions have disrupted self-control (Baler and Volkow, 2006). Therefore, urges for reward caused the individuals with low self-control to not be able to block this motivation to rewards. As a result, harmful consequences and risk behaviors resulted (Evans et al., 2003). As alluded to earlier, the high risk preference was induced by ineffectual self-control at the cognitive level, underlying intense motivation for reward. Correspondingly, individuals with low self-control, which means weak "cognitive control," had high tendencies toward risk behavior, owing to relative hyperactivity of the "socioemotional" system (Steinberg, 2008). Taken together, we postulated that the brain regions in the control network (i.e., the ACC and the prefrontal cortex) and "socioemotional" system (i.e., the OFC) could account for the relationship between self-control and risk preference.

In the present study, we sought to explore the neural substrates underlying the association between self-control and risk preference using voxel-based morphometry (VBM), in conjunction with the resting-state functional connectivity (RSFC) methods. Previous work has used this method to explore the brain morphometric and rs-fMRI functional connectivity in various brain disorders (Liao et al., 2011a,b). To our knowledge, there are notably few studies that have investigated the relationship between self-control and risk preference, based on combination of this two methods. The VBM can characterize the anatomical trait throughout the brain (Ashburner and Friston, 2000; Bellgrove et al., 2004). This method is also suitable to detect individual differences in cognitive control and personality (Spampinato et al., 2009). The RSFC is a technique used to explore the intrinsic functional architecture of the human brain, 
when subjects were not engaged in external tasks (Lewandowska et al., 2008). Intriguingly, it has been widely acknowledged that altered gray matter volumes (GMV) in some regions was ordinarily accompanied by corresponding changes of RSFCs (Gili et al., 2011). Whilst combining these two methods can drastically broaden our knowledge about the neural correlates of some behavior ( $\mathrm{Li}$ et al., 2012). Therefore, the Self-control Scale (SCS) and the Wheel of Fortune task (WOF) were used to assess individuals' ability of self-control and risk preference, respectively. In sample 1, we performed whole-brain VBM analyses to search the GMV of the regions correlated with risk preference. In sample 2, we defined the brain regions in which the GMV had exhibited significant correlation with risk preference in sample 1 as seed regions to calculate voxel-wise functional connectivity. The functional connectivity related with risk preference and self-control were, respectively examined. Finally, mediation analysis was performed to further explore whether RSFCs contributed to the relationship between selfcontrol and risk preference or not.

\section{MATERIALS AND METHODS}

\section{Participants and Procedure}

Ninety nine healthy college students were recruited as the sample 1 (47 males; age, $20.37 \pm 1.63$ years), whilst 90 healthy college students were enrolled as the independent sample 2 . Ten participants were excluded due to the excessive head movement [exceed framewise displacement (FD) $>0.2 \mathrm{~mm}$ ] during scanning (see details below), and 80 participants remained in sample 2 ( 33 males; age, $20.33 \pm 1.83$ years). Each participant was right-handed and had normal or corrected-to-normal vision. No history of psychiatric disorder was reported as well. All participants gave the informed consent. Prior to MRI scanning, participants were required to finish the self-control scale and the WOF. Afterward, they were reimbursed for participation.

\section{Measures}

\section{The Self-Control Scale}

Self-control ability was measured by the SCS, which has been widely considered to be able to assess the multiple domains of individual self-control (Tangney et al., 2004). Specifically, 36 items are included in this scale and can be divided into 5 dimensions, including general self-discipline (11 items), impulsive control (10 items), healthy habit (7 items), work/study ethic (4 items), and reliability ( 4 items). Each item is rated on the rank from 1 (strongly disagree) to 5 (strongly agree) (e.g., "I am good at resisting temptation"). The score of negative wording items was reversed so that the higher total score can represent the better self-control ability. Self-control scores in the present study were normally distributed (Kolmogorov-Smirnov $z=0.810, p=0.528)$. This scale showed a good internal consistency $(\alpha=0.89)$ (Tangney et al., 2004).

\section{The Wheel of Fortune Task}

In this study, the risk preference was assessed via the WOF (Ernst et al., 2004), in which participants indicated their preference between a certain option and a risk option for monetary rewards. The certain options were rewarded with fixed $¥ 1$, and the risk options comprised combinations of 19 probabilities (5 to 95\% with an interval of $5 \%$ ) and 9 monetary amounts ( $¥ 1$ to 9; with an interval of $¥ 1$; averaged $¥ 5$ ), yeilding a total of 171 trials (19 probabilities $\times 9$ monetary amounts) in one session. During each trial, if risky option was chosen, a blue dot would stop either in green (indicating win) or red area (indicating they earned nothing) of the wheel as a feedback. Alternatively, if the certain option was chosen, the participant would surely acquire the fixed rewards (see Figure 1). Each subject was informed that their payment would be the monetary payment they got during the whole task. Of note, the expected value of rewards can affect individuals' choice. Therefore, the expected value of rewards in the fixed option and risky option were equivalently designed (Kahneman and Tversky, 2013). In line with previous study, the gambling rate was obtained from the ratio of risky choice selected in all trials. Ultimately, the gambling rate was used to measure risk preference (Ernst et al., 2004). The higher gambling rate indicates higher risk tolerance.

\section{fMRI Acquisition}

The structural MRI and resting-state fMRI scans were obtained with a Siemens 3T scanner (Siemens MAGNETOM Trio TIM, Erlangen, Germany). The magnetization prepared rapid acquisition gradient-echo (MPRAGE) sequence $(128$ slices; $\mathrm{TR}=2530 \mathrm{~ms} ; \mathrm{TE}=3.39 \mathrm{~ms}$; flip angle $=7^{\circ} ; 256 \times 256$ matrix) was used to acquire high-resolution T1-weighted anatomical images (voxel size $=1 \mathrm{~mm}^{3} \times 1 \mathrm{~mm}^{3} \times 1.33 \mathrm{~mm}^{3}$ ). Resting-state fMRI images were acquired using $\mathrm{T} 2{ }^{*}$-weighted Echo Planar Imaging (EPI) sequence $\left(\mathrm{TR}=2000 \mathrm{~ms}, \mathrm{TE}=30 \mathrm{~ms}\right.$, flip angle $=90^{\circ}$, resolution matrix $=64 \times 64, \mathrm{FOV}=200 \mathrm{~mm}^{2} \times 200 \mathrm{~mm}^{2}, 33$ slices, voxel size $=3.1 \mathrm{~mm} \times 3.1 \mathrm{~mm} \times 3.6 \mathrm{~mm}$ ). Participants were required to keep their eyes closed without sleeping and thinking during the scan. These scans lasted for $8 \mathrm{~min}$, and incorporated 240 volumes.

\section{Date Analyses VBM Analyses}

Voxel-based morphometry analyses were implemented via SPM12, in conjunction with DARTEL and vbm8 toolbox ${ }^{1}$.

\section{Preprocessing}

Before VBM analyses, the SPM12 was used to display each MRI image for checking whether there were artifacts and gross anatomical abnormalities. As indicated by previous guidance (Ashburner, 2007), all these structural images were manually adjusted to make the anterior commissar match the origin $(0$, $0,0)$, and 3-dimensional Montreal Neurological Institute (MNI) space primarily. Then, these reoriented images were segmented into gray matter, white matter and cerebrospinal fluid. Afterward, the versions of the gray and white matter tissues imported by DARTEL were used to generate the flow fields and a series of template images. Subsequently, those obtained images were

\footnotetext{
${ }^{1}$ http://www.fil.ion.ucl.ac.uk/spm/software/spm12/
} 


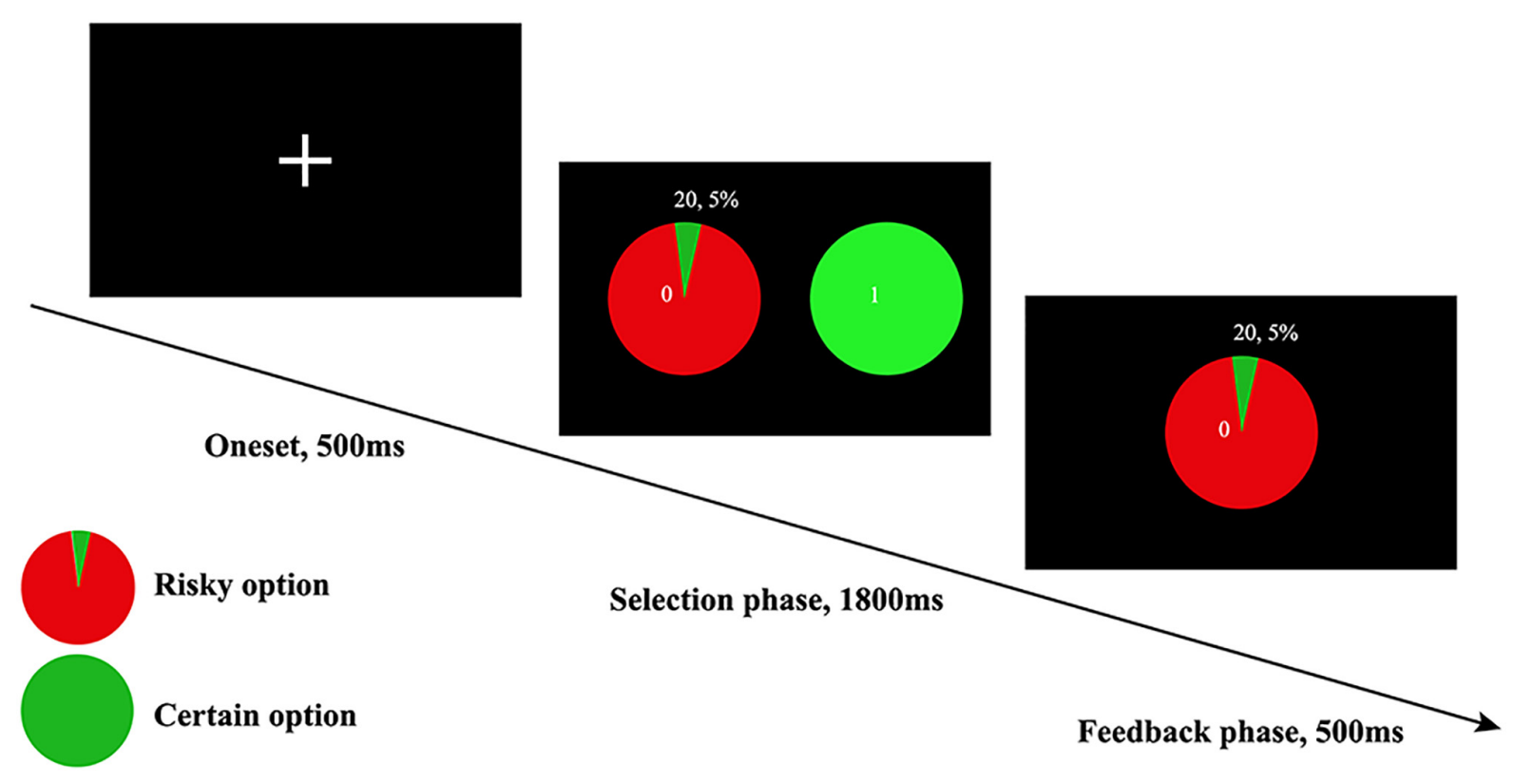

FIGURE 1 | The WOF task, only the winning version was displayed here, which meant when an individual chose to accept the risky choice, then he or she would get $¥ 20$ as feedback. The trial-by-trial feedback was real in accordance to the probabilities presented, and participants’ payment was based on their performance on all trials.

smoothed with $8 \mathrm{~mm}$ Gaussian FWHM, modulated, and spatially normalized to create Jacobian scaled GM images resliced to $2 \mathrm{~mm} \times 2 \mathrm{~mm} \times 2 \mathrm{~mm}$ voxel size in MNI space.

\section{Second level modeling analyses}

The multiple linear regression was performed to capture the brain regions that were correlated with risk preference in sample 1 (99 participants). In this model, the gambling rate was defined as a covariate of interest, whilst the age and gender were included as covariates of no interest according to previous findings (Kulynych et al., 1994; Good et al., 2001). Afterward, the mask with absolute threshold of 0.2 was performed to restrict the gray matter areas (Guo et al., 2017). Then, the MATLAB script "get_totals"2 was used to extract the regional GMV. Eventually, T contrasts were applied to explore the voxels that correlated with risk preference with a threshold at $p<0.001$. The final results were corrected by small volume correction (Sphere at peak MNI; radius of $\mathrm{VOI}=20 \mathrm{~mm}$ ).

\section{RSFC Analyses}

\section{Preprocessing}

The rs-fMRI images were preprocessed in the DPARSF toolbox ${ }^{3}$ (Yan and Zang, 2010). Firstly, to preserve from the distortion magnetization disequilibrium and the participant's adaptation to the scanning noise, the first 10 volumes of each participant were discarded. The remaining 230 volumes needed to be corrected for temporal shifts between slices and correcting for motion. Following this, all realigned images were normalized to the MNI

${ }^{2}$ http://www.cs.ucl.ac.uk/staff/g.ridgway/vbm/get_totals.m

${ }^{3}$ http://rfmri.org/dpabi template in $3 \mathrm{~mm}^{3} \times 3 \mathrm{~mm}^{3} \times 3 \mathrm{~mm}^{3}$, and smoothed with an isotropic $4 \mathrm{~mm}$ FWHM Gaussian kernel. To reduce the impact of head movement and nuisance signals, the white matter signal, cerebrospinal fluid signal (CFS), global signal, and head motion data were regressed out (Birn et al., 2006; Auer, 2008; Fox et al., 2009). Then, the temporal filtering $(0.01-0.08 \mathrm{~Hz})$ and detrending were performed to obtain low-frequency fluctuation from resting state $\mathrm{fMRI}$ data. Given the motion-related signal in resting-state fMRI data cannot be fully removed by regression of motion estimates (Power et al., 2012; Yan et al., 2013), the frame-wise motion censoring was performed. The threshold of FD $>0.2 \mathrm{~mm}$ as well as 1 back and 2 forward neighbors were used to remove volumes (Power et al., 2013). Motion censoring may result in a large number of eliminated volumes and too few remaining volumes that can lead to unreliable results. On this account, a 5-min criterion was set. Accordingly, 10 participants who had less than $5 \mathrm{~min}$ data remaining after censoring were excluded (Power et al., 2013). The mean FD for each participant was further regressed out at the group-level analyses.

\section{Functional connectivity analyses}

In functional connectivity analyses, the right orbitofrontal cortex (rOFC) derived from the outcomes in sample 1 were identified as seed region to calculate the whole-brain functional connectivity maps in the independent sample 2. Similarly, The functional connectivity was calculated in sample 2 based on seed region of the right posterior parietal cortex (rPPC). In order to produce functional connectivity maps, the BOLD time course from seed regions were extracted, and correlation of time course between each seed regions and the BOLD of all other brain voxels were computed. Then, all maps were transformed with the Fisher 

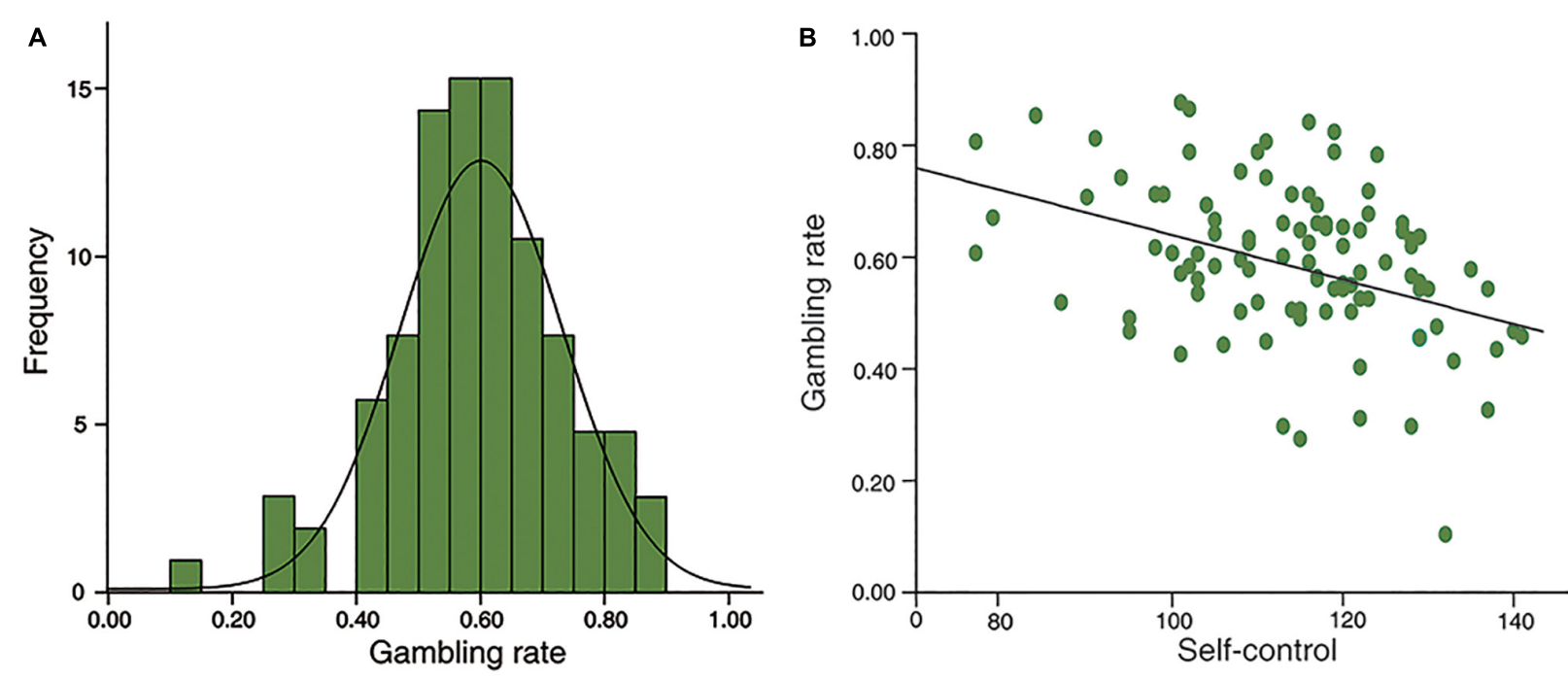

FIGURE 2 | Behavioral results. (A), the distributions of risk preference in sample 1; (B), self-control was negatively correlated with risk preference significantly $(r=-0.397, p<0.001)$.

$z$-values. To further determine the relations between functional connectivity of seed regions and risk preference, we performed the correlation analyses between this $z$-valued functional connectivity maps and individual's gambling rate. The regions from correlation analyses $(p<0.05$, AlphaSim correction, and cluster size $\geq 187$ ) were saved as ROIs for subsequent analyses. Afterward, to explore the relationship between self-control and this functional connectivity correlated with risk preference, we calculated the correlations between self-control and the connectivity values which were extracted from the seed regions' connectivity map of each participant. Finally, the mediation analysis was performed to explore the influence of this functional connectivity on the effect of self-control on risk preference.

\section{RESULTS}

\section{Behavioral Results}

The distribution is normal in self-control (Kolmogorov-Smirnov $z=0.810, p=0.528$ ) and gambling rate (Smirnov $z=0.588$, $p=0.880$; see Figure 2A). Furthermore, no gender difference was found in self-control $(t=1.567, d f=97, p=0.120)$, or in gambling rate $(t=1.115, d f=97, p=0.268)$. In addition, there were no significant correlations between age and self-control $(r=0.174$, $p=0.086)$, or gambling rate $(r=-0.107, p=0.293)$. Moreover, in line with previous studies, self-control scores were negatively associated with gambling rate $(r=-0.397, p<0.001$; Figure 2B).

\section{Neuroanatomical Correlates of Risk Preference}

To examine the neural substrates underlying risk preference, whole-brain VBM analyses were performed in sample 1. The results demonstrated that the GMV in rOFC (MNI peak coordinates: $18,50,-22$; voxels $=346 ; p<0.001$, small volume correction; see Figure 3A and Table 1) and the rPPC (MNI peak coordinates: $36,-72,42$; voxels $=119 ; p<0.001$, small volume correction; see Figure 3B and Table 1) were negatively associated with gambling rate. These results suggested that the GMV of rOFC and rPPC might be the underlying neuroanatomical basis of risk preference.

\section{RSFC Results}

Previous studies have demonstrated that the altered GMV in brain regions were accompanied by the altered functional coupling between these altered regions with other related regions (Lui et al., 2009; Gili et al., 2011). Consequently, we firstly investigated whether risk preference would be predicted by functional connectivity with regions (rOFC, MNI: 18, 50, -22 ; rPPC, MNI: 36, -72, 42) from the VBM results. The results showed that the gambling rate was positively correlated with functional connectivity between rOFC and right anterior cingulate cortex (rACC) under AlphaSim correction (see Figure 4 and Table 2). Notably, no other functional connectivity had a significant correlation with risk preference with the seed region of rPPC. Then, a correlational analysis was conducted to examine the relationship between self-control and functional connectivity of rOFC-rACC. The result demonstrated that the functional connectivity of rOFC-rACC was negatively correlated with selfcontrol scores $(r=-0.320, p=0.004)$.

\section{The Mediation Analysis}

To investigate how the functional connectivity of rOFC-rACC contributed to the effect of self-control on risk preference, mediation analysis using the INDIRECT procedure (Preacher and Hayes, 2008; Hayes and Scharkow, 2013) with 5000 bootstrap samples in SPSS (Statistical Product and Service Solutions) was performed. The estimate of the mediated effect $a^{*} b / c=0.370$, and the $95 \%$ confidence for intervals of 0.031 and 0.235 , suggesting 


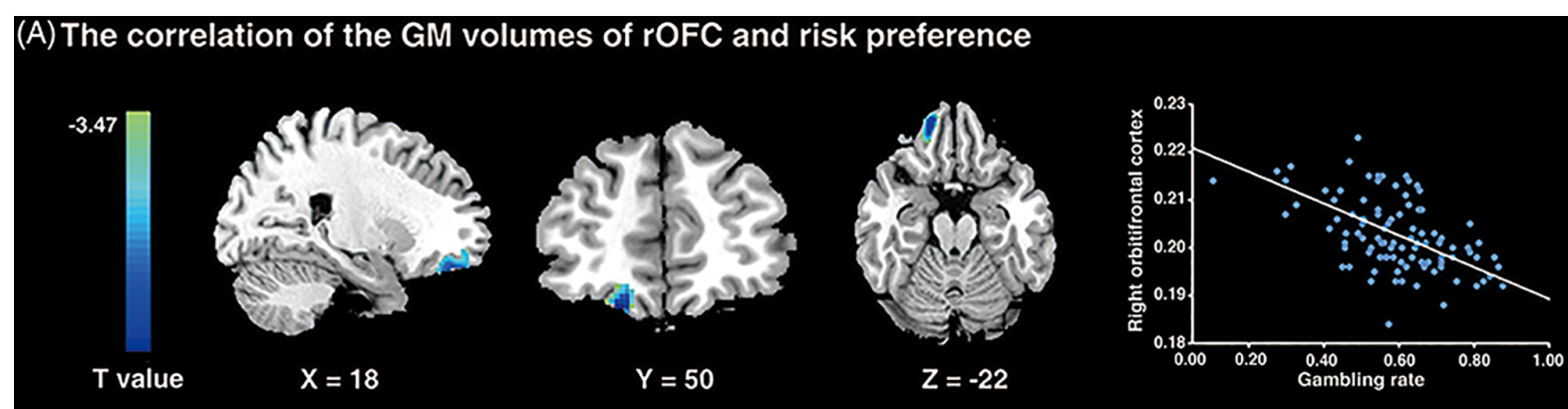

(B) The correlation of the GM volumes of rPPC and risk preference

$-3.47$

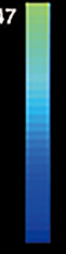

T value

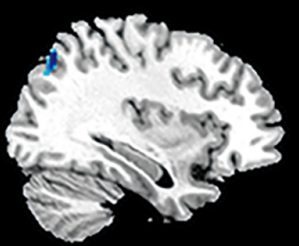

$X=36$

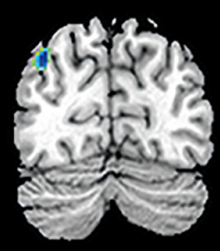

$Y=-72$

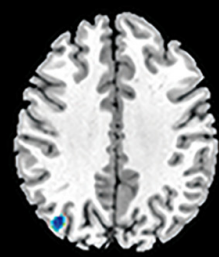

$Z=42$

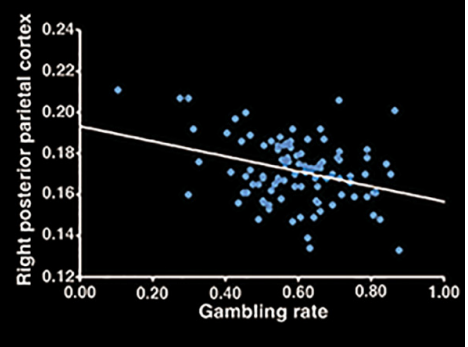

FIGURE 3 | VBM results. (A) The gambling rates were negatively correlated with the GM volumes in the right orbitofrontal cortex $(p<0.001$; small volume corrected). (B) The similar correlation was found between risk preference and the GM volumes of the right posterior parietal cortex ( $p<0.001$; small volume corrected). The scatter plots on the right are presented for visualization and not for statistical inference.

TABLE 1 | Areas of brain structures significantly correlated with risk preference only -: the brain regions negatively correlated with risk preference ( $p<0.001$; corrected).

\begin{tabular}{lccc}
\hline Brain regions & MNI & Voxels & T \\
\hline Only - & & & \\
R. orbitofrontal cortex & $18,50,-22$ & 346 & -4.18 \\
R. posterior parietal lobe & $36,-72,42$ & 119 & -3.43 \\
\hline
\end{tabular}

that $37 \%$ of the prediction of risk preference by functional connection of rOFC-rACC may have been mediated by selfcontrol (see Figure 5). This result indicated that the functional coupling of the rOFC-rACC might be crucially responsible for the effect of self-control on risk preference.

\section{DISCUSSION}

The present study investigated the neural substrates underlying the effect of self-control on risk preference by combining the VBM and RSFC analyses. In line with previous behavioral studies, the score of self-control was negatively associated with risk preference. The VBM results showed that the GMV of both rOFC and rPPC were negatively correlated with gambling rate. Moreover, increased connectivity between $\mathrm{rOFC}$ and $\mathrm{rACC}$ was positively correlated with more gambling rate. Finally, the mediation analysis revealed that self-control robustly mediated the effect of functional connectivity of rOFC-rACC on risk preference. Taken together, those findings provide a novel insight into the neural substrates accounting for the association between self-control and risk preference, and extend our understanding on risk preference.

In line with previous research, the scores of self-control were negatively correlated with gambling rate. A considerable number of studies have found that inadequate self-control can result in more maladaptive behaviors, such as addiction (Bechara, 2005; Fox et al., 2008; Bickel et al., 2012), impulsive buying (Rose, 2007; Vohs and Faber, 2007). Furthermore, straightforward studies on the relationship between self-control and risk preference also found that lacking of self-control could lead to more risky activities (Wulfert et al., 1999; Martins et al., 2004; Freeman and Muraven, 2010; Friehe and Schildberg-Hörisch, 2014). Therefore, in accordance with prior observations, our behavioral results revealed an intimate relationship between self-control and risk preference.

The whole-brain VBM analyses demonstrated that the GMV of the $\mathrm{rOFC}$ and $\mathrm{rPPC}$ were inversely correlated with risk preference, indicating that individuals with less GMV in these regions might have increased the preference for risk. In general, quite a few studies have found that lesions in the parietal cortex impede real-time updating of the probability of winning in gambling. Therefore, this financially challenged adjustment might lead to the experience of financial loss (Studer et al., 2015). Functional neuroimaging studies have highlighted that elevated activation of the parietal cortex assesses high risk in decision making (Symmonds et al., 2011; Studer et al., 2013). More remarkably, one robust study proved that the GMV of rPPC can predict individuals' risk preference (Gilaie-Dotan et al., 


\section{The functional connectivity of rOFC-rACC}
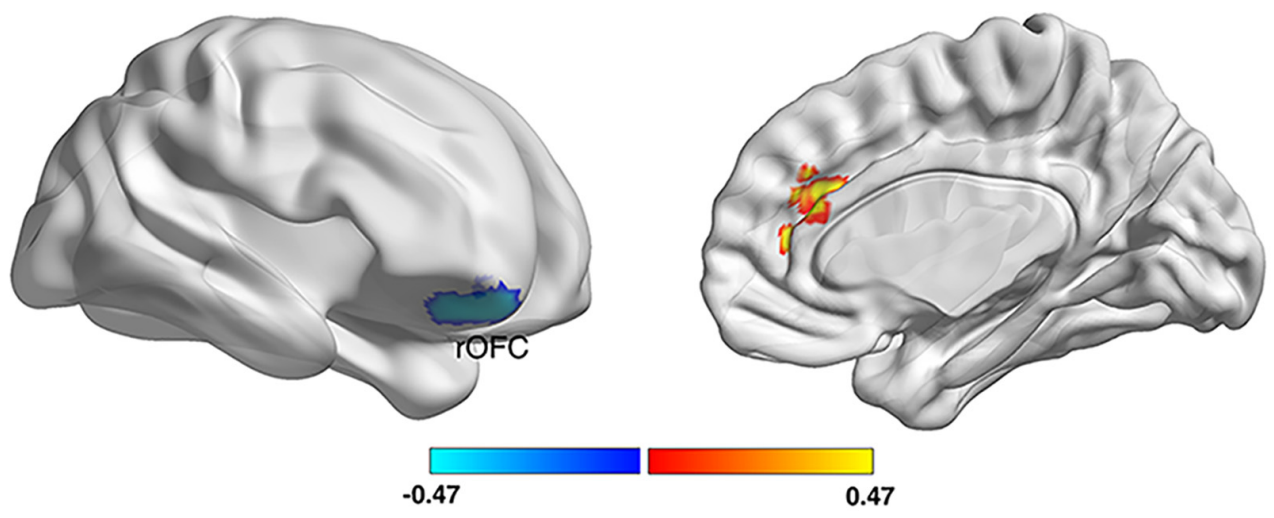

FIGURE 4 | Resting-state functional connectivity result. We defined the rOFC and the rPPC bases on the VBM analyses, respectively, as masks. Functional connectivity between seed regions and $\mathrm{rACC}$ was positively correlated with gambling rates $(p<0.05$; Alphasim corrected, cluster size $>187)$.

TABLE 2 | Functional connectivity correlated with risk preference $(p<0.05$, Alphasim corrected; rOFC, cluster size >187).

\begin{tabular}{|c|c|c|c|c|c|}
\hline Seed & Region & BA & Voxels & MNI & Correlation coefficient \\
\hline $\begin{array}{l}\text { R. orbitofrontal } \\
\text { cortex }\end{array}$ & $\begin{array}{l}\text { R. anterior } \\
\text { cingulate cortex }\end{array}$ & 32 & 320 & $15,33,9$ & 0.327 \\
\hline
\end{tabular}

2014). Thus, these studies demonstrably underscored that the parietal cortex made a critical contribution to risk preference. It is worthwhile to note that the OFC was involved in risky decisionmaking (Fukui et al., 2005; Jentsch et al., 2010). Increasing uncertainty of choice, which is ordinarily involved in risk, could induce the activation of OFC (Critchley et al., 2001). Specifically, the evaluation of the value of rewards in risk decision-making was consistently mediated by activation of the OFC (Ernst et al., 2002; Akitsuki et al., 2003). Additionally, it was confirmed that less GMV of the OFC was associated with more risky behaviors (Matsuo et al., 2009; Peper et al., 2013). These studies suggested that the OFC played a critical role in reward evaluation during risk decision making. The VBM method can predict individual differences in cognitive processes by comparing a voxel-wise of GMV, or neuronal density, and has been increasingly applied in pathological research (Cousijn et al., 2012). Previous evidence has suggested that reduced GMV might be expressed by reduced neuron density, which impedes the transmission efficiency of cognitive processes (Kanai and Rees, 2011). Accordingly, we interpret the decrease in GMV of PPC and OFC as limiting the comprehensive evaluation of risk levels and rewards, due to the inefficient information process. In brief, the rOFC and rPPC could be considered as the neuroanatomical substrates of risk preference.

In order to examine the functional neural substrates of the relationship between self-control and risk preference, the functional connectivity analyses were conducted. The results indicated that the enhanced coupling of the rOFC and rACC was positively correlated with the gambling rate. Many studies

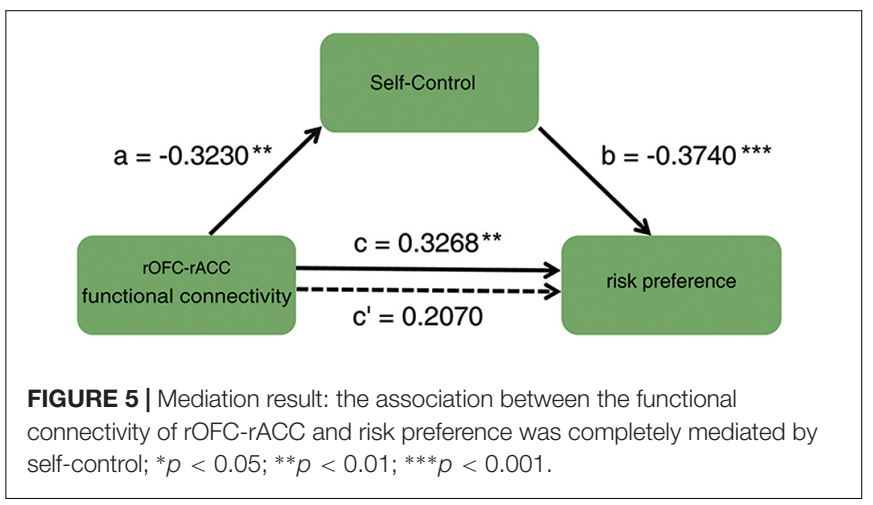

have provided convincing evidence that increased rACC activity was related to a lower probability of options in decision making (Elliot, 1999; Koechlin et al., 2000; Volz et al., 2003, 2005) or uncertain conditions that were generally contained in risk choice (Rao and Davim, 2008; Hewig et al., 2009; Payzan-LeNestour et al., 2013). Moreover, the risky choices were accompanied by increased activity in the ACC, which monitored the potential conflicts (Blair et al., 2006). Furthermore, some pathological studies found that patients with OFC damage were insensitive to differing risk conditions (Bechara et al., 2000; Hsu et al., 2005). Specifically, the OFC was widely observed to be involved in processing the subjective value of rewards, suggesting that there is a close relationship between the OFC and risk preference (Levy and Glimcher, 2011; Lin et al., 2012). More importantly, there were the greater activations of the ACC and OFC exhibited when individuals were making relatively high-risk decisions (Ernst et al., 2004; Rogers et al., 2004). It is paramount to note that elevated activation in the ACC is associated with tension between reward seeking and loss avoidance. In other words, the elevated activity in the ACC can be viewed as a state of conflict (Paulus et al., 2001), resulted from the preference for reward under high-risk circumstances (Rao and Davim, 
2008; Hewig et al., 2009). Considering the above, the OFC and ACC may serve reward seeking and conflict monitoring functions. The functional connectivity between the rOFC and rACC might signal the pursuit of larger reinforcers, which can result in hyperactivation of the "socioemotional" system. Nevertheless, activation of the rACC in the "cognitive control" system only monitors conflict in decision making but cannot successfully override hyperactivation of "socioemotional" system. Consequently, the enhanced functional connectivity of the rOFCrACC may be involved in the intense conflict of reward seeking, which gives rise to high risk preference. The RSFC is essential for individuals with high risk preferences. Collectively, our RSFC results indicated that the functional connectivity of the rOFCrACC might be the neural representation of risk preference.

Importantly, the mediation analysis identified that the impact of the rOFC-rACC functional connectivity on risk preference was robustly mediated by self-control. Notably, it has been alluded that the OFC evaluated rewards in risky decision making (Ernst et al., 2002; Akitsuki et al., 2003), and that the rACC monitored the state of conflict construed by reward seeking and loss avoidance (Carter et al., 1998; Botvinick et al., 2004; Ridderinkhof et al., 2004; Blair et al., 2006). These findings indicated that the functional connectivity of rOFCrACC was a signal of pursuing high-value rewards. Moreover, high sensitivity for rewards showed an inclination to high risk preference (Leiserson and Pihl, 2007). However, in favor of loss avoidance, strong self-control ability can restrain the desire for obtaining rewards, which triggers activation of the "socioemotional" system. Such a restraint might restrain reward seeking elicited by the activation of the OFC and ACC (Jimura et al., 2013). In contrast, low self-control, or the relatively weak activity of the "cognitive control" system, led to an inability to resist irrational reward seeking. Accordingly, heightened activity of the "socioemotional" system compelled individuals toward risky activity (Steinberg et al., 2015). In brief, the clue of monetary reward activated the OFC and ACC. Once these brain regions are activated, it would motivate individuals to pursuit a reward that might result in a harmful consequence and selfcontrol failure. At this point, the more adequate self-control will be needed to resist this motivation which may cause risk reference. Therefore, risk behaviors was the fruit of the failure of self-control induced by reward purchasing. Taken together, the functional connectivity of rOFC-rACC might present the neural substrates responsible for the relationship between self-control and risk preference.

However, the present study inevitably has some limitations. Primarily, the self-control ability was assessed by a scale, which is less effective than a behavioral task. It is advocated that a behavioral task can be used to estimate the self-control ability under sufficient conditions. It is noteworthy that trait self-control is relatively malleable (Bergen et al., 2012). In the future, it would shed light on holding definite promise for ameliorating the problem of risky behavior, if a reliable way can be found to help individuals with high risk preference to increase their self-control strength. As a second limitation, this study is just a correlative study, which is not able to draw a causal conclusion. Thus, future research can use more experimental methods, such as task fMRI method, to explore the causal effect of self-control on risk preference in depth.

In conclusion, the present investigation showed that selfcontrol was negatively associated with risk preference, suggesting that inadequate self-control ability is associated with higher risk preference. Furthermore, the VBM analyses indicated that the GMV of the rOFC and rPPC were negatively correlated with risk preference. These results demonstrated that the rOFC and rPPC might be the neuroanatomical correlations of risk preference. Moreover, the RSFC results indicated that the functional connectivity of rOFC-rACC was positively correlated with risk preference. We further identified the completely mediating role of self-control in the relationship between these functional connectivity and risk preference. Overall, our study provides a novel insight into the neural substrates underlying the relationship between self-control and risk preference, and extends our understanding on risk preference.

\section{DATA AVAILABILITY}

The datasets for this manuscript are not publicly available because the datasets the privacy information of the subjects, and a confidentiality agreement has been signed to protect this. Requests to access the data sets should be directed to the corresponding author, TF, fengty0@swu.edu.cn.

\section{ETHICS STATEMENT}

This study was carried out in accordance with the recommendations of Southwest University institutional review board with written informed consent from all subjects. All subjects gave written informed consent in accordance with the Declaration of Helsinki. The protocol was approved by the Southwest University Institutional Review Board.

\section{AUTHOR CONTRIBUTIONS}

MW, ZC, SZ, RZ, and TX designed the study and collected and analyzed the data. MW, TS, and TF wrote the manuscript. TF and TS revised the manuscript. All authors approved the version to be published.

\section{FUNDING}

This study was supported by the National Natural Science Foundation of China (31571128 and 31400908), the Philosophy and Social Science Foundation of Henan Province, China (2015BJY007), and the Henan University Philosophy and Social Science Innovation Team (2019CXTD009).

\section{ACKNOWLEDGMENTS}

We thank Dr. Changming Chen and Dr. Lei Liu for assistance with language. 


\section{REFERENCES}

Akitsuki, Y., Sugiura, M., Watanabe, J., Yamashita, K., Sassa, Y., Awata, S., et al. (2003). Context-dependent cortical activation in response to financial reward and penalty: an event-related fMRI study. Neuroimage 19, 1674-1685. doi: 10.1016/S1053-8119(03)00250-7

Angkinand, A., and Wihlborg, C. (2010). Deposit insurance coverage, ownership, and banks' risk-taking in emerging markets. J. Int. Money Finance 29, 252-274. doi: 10.1016/j.jimonfin.2009.08.001

Arana, F. S., Parkinson, J. A., Hinton, E., Holland, A. J., Owen, A. M., and Roberts, A. C. (2003). Dissociable contributions of the human amygdala and orbitofrontal cortex to incentive motivation and goal selection. J. Neurosci. 23, 9632-9638. doi: 10.1523/JNEUROSCI.23-29-09632.2003

Ashburner, J. (2007). A fast diffeomorphic image registration algorithm. Neuroimage 38, 95-113. doi: 10.1016/j.neuroimage.2007.07.007

Ashburner, J., and Friston, K. J. (2000). Voxel-based morphometry-the methods. Neuroimage 11, 805-821. doi: 10.1006/nimg.2000.0582

Auer, D. P. (2008). Spontaneous low-frequency blood oxygenation level-dependent fluctuations and functional connectivity analysis of the 'resting'brain. Magn. Reson. Imaging 26, 1055-1064. doi: 10.1016/j.mri.2008.05.008

Baler, R. D., and Volkow, N. D. (2006). Drug addiction: the neurobiology of disrupted self-control. Trends Mol. Med. 12, 559-566. doi: 10.1016/j.molmed. 2006.10.005

Bechara, A. (2005). Decision making, impulse control and loss of willpower to resist drugs: a neurocognitive perspective. Nat. Neurosci. 8:1458. doi: 10.1038/nn1584

Bechara, A., Damasio, H., and Damasio, A. R. (2000). Emotion, decision making and the orbitofrontal cortex. Cerebral Cortex 10, 295-307. doi: 10.1093/cercor/ 10.3.295

Bellgrove, M. A., Hester, R., and Garavan, H. (2004). The functional neuroanatomical correlates of response variability: evidence from a response inhibition task. Neuropsychologia 42, 1910-1916. doi: 10.1016/j.neuropsychologia.2004.05.007

Bergen, A. E., Newbyclark, I. R., and Brown, A. (2012). Low trait self-control in problem gamblers: evidence from self-report and behavioral measures. J. Gambl. Stud. 28, 637-648. doi: 10.1007/s10899-011-9274-9

Bickel, W. K., Jarmolowicz, D. P., Mueller, E. T., Gatchalian, K. M., and McClure, S. M. (2012). Are executive function and impulsivity antipodes? A conceptual reconstruction with special reference to addiction. Psychopharmacology 221, 361-387. doi: 10.1007/s00213-012-2689-x

Birn, R. M., Diamond, J. B., Smith, M. A., and Bandettini, P. A. (2006). Separating respiratory-variation-related fluctuations from neuronal-activityrelated fluctuations in fMRI. Neuroimage 31, 1536-1548. doi: 10.1016/j. neuroimage.2006.02.048

Blair, K., Marsh, A. A., Morton, J., Vythilingam, M., Jones, M., Mondillo, K., et al. (2006). Choosing the lesser of two evils, the better of two goods: specifying the roles of ventromedial prefrontal cortex and dorsal anterior cingulate in object choice. J. Neurosci. 26, 11379-11386. doi: 10.1523/JNEUROSCI.1640-06.2006

Botvinick, M. M., Cohen, J. D., and Carter, C. S. (2004). Conflict monitoring and anterior cingulate cortex: an update. Trends Cogn. Sci. 8, 539-546. doi: $10.1016 /$ j.tics.2004.10.003

Breiter, H. C., Aharon, I., Kahneman, D., Dale, A., and Shizgal, P. (2001). Functional imaging of neural responses to expectancy and experience of monetary gains and losses. Neuron 30, 619-639. doi: 10.1016/S0896-6273(01) 00303-8

Breiter, H. C., Rauch, S. L., Kwong, K. K., Baker, J. R., Weisskoff, R. M., Kennedy, D. N., et al. (1996). Functional magnetic resonance imaging of symptom provocation in obsessive-compulsive disorder. Arch. Gen. Psychiatry 53, 595606. doi: 10.1001/archpsyc.1996.01830070041008

Carter, C. S., Braver, T. S., Barch, D. M., Botvinick, M. M., Noll, D., and Cohen, J. D. (1998). Anterior cingulate cortex, error detection, and the online monitoring of performance. Science 280, 747-749. doi: 10.1126/science.280.5364.747

Carver, C. S., and Scheier, M. F. (2012). Attention and Self-Regulation: A ControlTheory Approach to Human Behavior. Berlin: Springer Science \& Business Media.

Casey, B. J., Getz, S., and Galvan, A. (2008). The adolescent brain. Dev. Rev. 28, 62-77. doi: 10.1016/j.dr.2007.08.003

Christopoulos, G. I., Tobler, P. N., Bossaerts, P., Dolan, R. J., and Schultz, W. (2009). Neural correlates of value, risk, and risk aversion contributing to decision making under risk. J. Neurosci. 29, 12574-12583. doi: 10.1523/ JNEUROSCI.2614-09.2009

Cohen, J. D. (2005). The vulcanization of the human brain: a neural perspective on interactions between cognition and emotion. J. Econ. Perspect. 19, 3-24. doi: $10.1257 / 089533005775196750$

Cousijn, J., Wiers, R. W., Ridderinkhof, K. R., van den Brink, W., Veltman, D. J., and Goudriaan, A. E. (2012). Grey matter alterations associated with cannabis use: results of a VBM study in heavy cannabis users and healthy controls. Neuroimage 59, 3845-3851. doi: 10.1016/j.neuroimage.2011.09.046

Critchley, H. D., Mathias, C. J., and Dolan, R. J. (2001). Neural activity in the human brain relating to uncertainty and arousal during anticipation. Neuron 29, 537-545. doi: 10.1016/S0896-6273(01)00225-2

de Ridder, D. T., Lensveltmulders, G., Finkenauer, C., Stok, F. M., and Baumeister, R. F. (2012). Taking stock of self-control: a meta-analysis of how trait selfcontrol relates to a wide range of behaviors. Pers. Psychol. Rev. 16, 76-99. doi: 10.1177/1088868311418749

Donohew, L., Zimmerman, R., Cupp, P. S., Novak, S., Colon, S., and Abell, R. (2000). Sensation seeking, impulsive decision-making, and risky sex: implications for risk-taking and design of interventions. Pers. Individ. Diff. 28, 1079-1091. doi: 10.1016/S0191-8869(99)00158-0

Elliot, A. J. (1999). Approach and avoidance motivation and achievement goals. Educ. Psychol. 34, 169-189. doi: 10.1207/s15326985ep3403_3

Engelmann, J. B., and Tamir, D. (2009). Individual differences in risk preference predict neural responses during financial decision-making. Brain Res. 1290, 28-51. doi: 10.1016/j.brainres.2009.06.078

Ernst, M., Bolla, K., Mouratidis, M., Contoreggi, C., Matochik, J. A., Kurian, V., et al. (2002). Decision-making in a risk-taking task: a PET study. Neuropsychopharmacology 26, 682-691. doi: 10.1016/S0893-133X(01)00414-6

Ernst, M., Nelson, E. E., McClure, E. B., Monk, C. S., Munson, S., Eshel, N., et al. (2004). Choice selection and reward anticipation: an fMRI study. Neuropsychologia 42, 1585-1597. doi: 10.1016/j.neuropsychologia.2004.05.011

Evans, J. L., Hahn, J. A., Page-Shafer, K., Lum, P. J., Stein, E. S., Davidson, P. J., et al. (2003). Gender differences in sexual and injection risk behavior among active young injection drug users in San Francisco (the UFO Study). J. Urban Health 80, 137-146. doi: 10.1093/jurban/jtg137

Fox, H., Hong, K., and Sinha, R. (2008). Difficulties in emotion regulation and impulse control in recently abstinent alcoholics compared with social drinkers. Addict. Behav. 33, 388-394. doi: 10.1016/j.addbeh.2007.10.002

Fox, M. D., Zhang, D., Snyder, A. Z., and Raichle, M. E. (2009). The global signal and observed anticorrelated resting state brain networks. J. Neurophysiol. 101, 3270-3283. doi: $10.1152 /$ jn. 90777.2008

Freeman, N., and Muraven, M. (2010). Self-control depletion leads to increased risk taking. Soc. Psychol. Pers. Sci. 1, 175-181. doi: 10.1177/194855060936 0421

Friehe, T., and Schildberg-Hörisch, H. (2014). Crime and Self-Control Revisited: Disentangling the Effect of Self-Control on Risk and Social Preferences. Available at: https://EconPapers.repec.org/RePEc:iza:izadps:dp8109.

Fukui, H., Murai, T., Fukuyama, H., Hayashi, T., and Hanakawa, T. (2005). Functional activity related to risk anticipation during performance of the Iowa gambling task. Neuroimage 24, 253-259. doi: 10.1016/j.neuroimage.2004.08.028

Galvan, A., Hare, T., Voss, H., Glover, G., and Casey, B. J. (2010). Risk-taking and the adolescent brain: who is at risk? Dev. Sci. 10, F8-F14.

Galvan, A., Hare, T. A., Parra, C. E., Penn, J., Voss, K., Glover, G., et al. (2006). Earlier development of the accumbens relative to orbitofrontal cortex might underlie risk-taking behavior in adolescents. J. Neurosci. 26:6885.

Gilaie-Dotan, S., Tymula, A., Cooper, N., Kable, J. W., Glimcher, P. W., and Levy, I. (2014). Neuroanatomy predicts individual risk attitudes. J. Neurosci. 34, 12394-12401. doi: 10.1523/JNEUROSCI.1600-14.2014

Gili, T., Cercignani, M., Serra, L., Perri, R., Giove, F., Maraviglia, B., et al. (2011). Regional brain atrophy and functional disconnection across Alzheimer's disease evolution. J. Neurol. Neurosurg. Psychiatry 82, 58-66. doi: 10.1136/jnnp.2009. 199935

Goldstein, R. Z., Alia-Klein, N., Tomasi, D., Zhang, L., Cottone, L. A., Maloney, T., et al. (2007). Is decreased prefrontal cortical sensitivity to monetary reward associated with impaired motivation and self-control in cocaine addiction? Am. J. Psychiatry 164, 43-51.

Good, C. D., Johnsrude, I. S., Ashburner, J., Henson, R. N., Friston, K. J., and Frackowiak, R. S. (2001). A voxel-based morphometric study of ageing in 465 
normal adult human brains. Neuroimage 14, 21-36. doi: 10.1006/nimg.2001. 0786

Gowin, J. L., Mackey, S., and Paulus, M. P. (2013). Altered risk-related processing in substance users: imbalance of pain and gain. Drug Alcohol Depend. 132, 13-21. doi: 10.1016/j.drugalcdep.2013.03.019

Guo, Y., Chen, Z., and Feng, T. (2017). Neural substrates underlying balanced time perspective: a combined voxel-based morphometry and resting-state functional connectivity study. Behav. Brain Res. 332, 237-242. doi: 10.1016/j.bbr.2017. 06.005

Han, S. D., Boyle, P. A., Konstantinos, A., Fleischman, D. A., Lei, Y., Edmonds, E. C., et al. (2012). Neural intrinsic connectivity networks associated with risk aversion in old age. Behav. Brain Res. 227, 233-240. doi: 10.1016/j.bbr.2011. 10.026

Harbison, H. S., Austin, E. L., Hook, E. W. III, and Muzny, C. A. (2018). Is there a continuum of risk for sexually transmitted infections among african american women? Sex Transm. Dis. 45, e20-e22. doi: 10.1097/OLQ.0000000000000750

Hayes, A. F., and Scharkow, M. (2013). The relative trustworthiness of inferential tests of the indirect effect in statistical mediation analysis: does method really matter? Psychol. Sci. 24, 1918-1927. doi: 10.1177/0956797613480187

Hewig, J., Straube, T., Trippe, R. H., Kretschmer, N., Hecht, H., Coles, M. G., et al. (2009). Decision-making under risk: an fMRI study. J. Cogn. Neurosci. 21, 1642-1652. doi: 10.1162/jocn.2009.21112

Hsu, M., Bhatt, M., Adolphs, R., Tranel, D., and Camerer, C. F. (2005). Neural systems responding to degrees of uncertainty in human decision-making. Science 310, 1680-1683. doi: 10.1126/science.1115327

Huettel, S. A., Song, A. W., and McCarthy, G. (2005). Decisions under uncertainty: probabilistic context influences activation of prefrontal and parietal cortices. J. Neurosci. 25, 3304-3311. doi: 10.1523/JNEUROSCI.5070-04.2005

Inzlicht, M., Schmeichel, B. J., and Macrae, C. N. (2014). Why self-control seems (but may not be) limited. Trends Cogn. Sci. 18, 127-133. doi: 10.1016/j.tics.2013. 12.009

Jentsch, J. D., Woods, J. A., Groman, S. M., and Seu, E. (2010). Behavioral characteristics and neural mechanisms mediating performance in a rodent version of the balloon analog risk task. Neuropsychopharmacology 35:1797. doi: $10.1038 /$ npp. 2010.47

Jimura, K., Chushak, M. S., and Braver, T. S. (2013). Impulsivity and self-control during intertemporal decision making linked to the neural dynamics of reward value representation. J. Neurosci. 33, 344-357. doi: 10.1523/JNEUROSCI.091912.2013

Kahneman, D., and Tversky, A. (2013). "Prospect theory: an analysis of decision under risk," in Handbook of the Fundamentals of Financial Decision Making: Part I, eds L. C. Maclean and W. T. Ziemba (Singapore: World Scientific), 99-127. doi: 10.1142/9789814417358_0006

Kanai, R., and Rees, G. (2011). The structural basis of inter-individual differences in human behaviour and cognition. Nat. Rev. Neurosci. 12, 231-242. doi: $10.1038 / \mathrm{nrn} 3000$

Knoch, D., and Fehr, E. (2010). Resisting the power of temptations: the right prefrontal cortex and self-control. Ann. N. Y. Acad. Sci. 1104, 123-134. doi: 10.1196/annals.1390.004

Koechlin, E., Corrado, G., Pietrini, P., and Grafman, J. (2000). Dissociating the role of the medial and lateral anterior prefrontal cortex in human planning. Proc. Natl. Acad. Sci. 97, 7651-7656. doi: 10.1073/pnas.130177397

Kool, W., McGuire, J. T., Wang, G. J., and Botvinick, M. M. (2013). Neural and behavioral evidence for an intrinsic cost of self-control. PLoS One 8:e72626. doi: 10.1371/journal.pone.0072626

Kruglanski, A. W. (2018). Speaking in General: Reflections on my Work. The Motivated Mind. Abingdon: Routledge. doi: 10.4324/9781315175867-1

Kulynych, J. J., Vladar, K., Jones, D. W., and Weinberger, D. R. (1994). Gender differences in the normal lateralization of the supratemporal cortex: MRI surface-rendering morphometry of Heschl's gyrus and the planum temporale. Cerebral Cortex 4, 107-118. doi: 10.1093/cercor/4.2.107

Leiserson, V., and Pihl, R. O. (2007). Reward-sensitivity, inhibition of rewardseeking, and dorsolateral prefrontal working memory function in problem gamblers not in treatment. J. Gambl. Stud. 23, 435-455. doi: 10.1007/s10899007-9065-5

Levy, D. J., and Glimcher, P. W. (2011). Comparing apples and oranges: using reward-specific and reward-general subjective value representation in the brain. J. Neurosci. 31, 14693-14707. doi: 10.1523/JNEUROSCI.2218-11.2011
Lewandowska, M., Bekisz, M., Szymaszek, A., Wrobel, A., and Szelag, E. (2008). Towards electrophysiological correlates of auditory perception of temporal order. Neurosci. Lett. 437, 139-143. doi: 10.1016/j.neulet.2008. 03.085

Li, R., Qin, W., Zhang, Y., Jiang, T., and Yu, C. (2012). The neuronal correlates of digits backward are revealed by voxel-based morphometry and resting-state functional connectivity analyses. PLoS One 7:e31877. doi: 10.1371/journal.pone. 0031877

Liao, W., Xu, Q., Mantini, D., Ding, J., Machado-de-Sousa, J. P., Hallak, J. E., et al. (2011a). Altered gray matter morphometry and resting-state functional and structural connectivity in social anxiety disorder. Brain Res. 1388, 167-177. doi: 10.1016/j.brainres.2011.03.018

Liao, W., Zhang, Z., Pan, Z., Mantini, D., Ding, J., Duan, X., et al. (2011b). Default mode network abnormalities in mesial temporal lobe epilepsy: a study combining fMRI and DTI. Hum. Brain Mapp. 32, 883-895. doi: 10.1002/hbm. 21076

Lin, A., Adolphs, R., and Rangel, A. (2012). Impaired learning of social compared to monetary rewards in autism. Front. Neurosci. 6:143. doi: 10.3389/fnins.2012. 00143

Lui, S., Deng, W., Huang, X., Jiang, L., Ma, X., Chen, H., et al. (2009). Association of cerebral deficits with clinical symptoms in antipsychotic-naive first-episode schizophrenia: an optimized voxel-based morphometry and resting state functional connectivity study. Am. J. Psychiatry 166, 196-205. doi: 10.1176/appi. ajp. 2008.08020183

Lusk, J. L., and Coble, K. H. (2005). Risk perceptions, risk preference, and acceptance of risky food. Am. J. Agric. Econ. 87, 393-405. doi: 10.1111/j.14678276.2005.00730.x

Martins, S. S., Tavares, H., da Silva Lobo, D. S., Galetti, A. M., and Gentil, V. (2004). Pathological gambling, gender, and risk-taking behaviors. Addict. Behav. 29, 1231-1235. doi: 10.1016/j.addbeh.2004.03.023

Mataix-Cols, D., Wooderson, S., Lawrence, N., Brammer, M. J., Speckens, A., and Phillips, M. L. (2004). Distinct neural correlates of washing, checking, and hoarding symptomdimensions in obsessive-compulsive disorder. Arch. Gen. Psychiatry 61, 564-576. doi: 10.1001/archpsyc.61.6.564

Matsuo, K., Nicoletti, M., Nemoto, K., Hatch, J. P., Peluso, M. A., Nery, F. G., et al. (2009). A voxel-based morphometry study of frontal gray matter correlates of impulsivity. Hum. Brain Mapp. 30, 1188-1195. doi: 10.1002/hbm. 20588

Mcclure, S. M., and Bickel, W. K. (2015). A dual-systems perspective on addiction: contributions from neuroimaging and cognitive training. Ann. N. Y. Acad. Sci. 1327, 62-78. doi: 10.1111/nyas.12561

Metcalfe, J., and Mischel, W. (1999). A hot/cool-system analysis of delay of gratification: dynamics of willpower. Psychol. Rev. 106:3. doi: 10.1037/0033295X.106.1.3

Mullins, J. W., and Forlani, D. (2005). Missing the boat or sinking the boat: a study of new venture decision making. J. Bus. Ventur. 20, 47-69. doi: 10.1016/j. jbusvent.2003.08.001

Paulus, M. P., Hozack, N., Zauscher, B., McDowell, J. E., Frank, L., Brown, G. G., et al. (2001). Prefrontal, parietal, and temporal cortex networks underlie decision-making in the presence of uncertainty. Neuroimage 13, 91-100. doi: 10.1006/nimg.2000.0667

Payzan-LeNestour, E., Dunne, S., Bossaerts, P., and O’Doherty, J. P. (2013). The neural representation of unexpected uncertainty during value-based decision making. Neuron 79, 191-201. doi: 10.1016/j.neuron.2013.04.037

Peper, J. S., Koolschijn, P. C. M., and Crone, E. A. (2013). Development of risk taking: contributions from adolescent testosterone and the orbito-frontal cortex. J. Cogn. Neurosci. 25, 2141-2150. doi: 10.1162/jocn_a_00445

Power, J. D., Barnes, K. A., Snyder, A. Z., Schlaggar, B. L., and Petersen, S. E. (2012). Spurious but systematic correlations in functional connectivity MRI networks arise from subject motion. Neuroimage 59, 2142-2154. doi: 10.1016/j. neuroimage.2011.10.018

Power, J. D., Barnes, K. A., Snyder, A. Z., Schlaggar, B. L., and Petersen, S. E. (2013). Steps toward optimizing motion artifact removal in functional connectivity MRI; a reply to Carp. Neuroimage 76, 439-441. doi: 10.1016/j.neuroimage.2012. 03.017

Preacher, K. J., and Hayes, A. F. (2008). Asymptotic and resampling strategies for assessing and comparing indirect effects in multiple mediator models. Behav. Res. Methods 40, 879-891. doi: 10.3758/BRM.40.3.879 
Rao, R. V., and Davim, J. (2008). A decision-making framework model for material selection using a combined multiple attribute decision-making method. Int. J. Adv. Manufact. Technol. 35, 751-760. doi: 10.1007/s00170-006-0752-7

Ridderinkhof, K. R., Van Den Wildenberg, W. P., Segalowitz, S. J., and Carter, C. S. (2004). Neurocognitive mechanisms of cognitive control: the role of prefrontal cortex in action selection, response inhibition, performance monitoring, and reward-based learning. Brain Cogn. 56, 129-140. doi: 10.1016/j.bandc.2004. 09.016

Robbins, R. N., and Bryan, A. (2004). Relationships between future orientation, impulsive sensation seeking, and risk behavior among adjudicated adolescents. J. Adolesc. Res. 19, 428-445. doi: 10.1177/0743558403258860

Rogers, R. D., Ramnani, N., Mackay, C., Wilson, J. L., Jezzard, P., Carter, C. S., et al. (2004). Distinct portions of anterior cingulate cortex and medial prefrontal cortex are activated by reward processing in separable phases of decisionmaking cognition. Biol. Psychiatry 55, 594-602. doi: 10.1016/j.biopsych.2003. 11.012

Rose, P. (2007). Mediators of the association between narcissism and compulsive buying: the roles of materialism and impulse control. Psychol. Addict. Behav. 21:576. doi: 10.1037/0893-164X.21.4.576

Ryan, K. K., MacKillop, J., and Carpenter, M. J. (2013). The relationship between impulsivity, risk-taking propensity and nicotine dependence among older adolescent smokers. Addict. Behav. 38, 1431-1434. doi: 10.1016/j.addbeh.2012. 08.013

Sitkin, S. B., and Pablo, A. L. (1992). Reconceptualizing the determinants of risk behavior. Acad. Manag. Rev. 17, 9-38. doi: 10.5465/amr.1992.4279564

Spampinato, M. V., Wood, J. N., De Simone, V., and Grafman, J. (2009). Neural correlates of anxiety in healthy volunteers: a voxel-based morphometry study. J. Neuropsychiatry Clin. Neurosci. 21, 199-205. doi: 10.1176/appi.neuropsych. 21.2.199

Steinberg, L. (2008). A social neuroscience perspective on adolescent risk-taking. Dev. Rev. 28, 78-106. doi: 10.1016/j.dr.2007.08.002

Steinberg, L., Oettingen, G., and Gollwitzer, P. M. (2015). The Neural Underpinnings of Adolescent Risk-Taking: The Roles of Reward-Seeking, Impulse Control, and Peers. Cambridge: Cambridge University Press, 173-192. doi: 10.1017/CBO9781139565790.009

Studer, B., Manes, F., Humphreys, G., Robbins, T. W., and Clark, L. (2013). Risksensitive decision-making in patients with posterior parietal and ventromedial prefrontal cortex injury. Cerebral Cortex 25, 1-9. doi: 10.1093/cercor/bht197

Studer, B., Manes, F., Humphreys, G., Robbins, T. W., and Clark, L. (2015). Risksensitive decision-making in patients with posterior parietal and ventromedial prefrontal cortex injury. Cerebral Cortex 25, 1-9. doi: 10.1093/cercor/bht197

Su, Y. S., Chen, J. T., Tang, Y. J., Yuan, S. Y., Mccarrey, A. C., and Goh, J. O. S. (2018). Age-related differences in striatal, medial temporal, and frontal involvement during value-based decision processing. Neurobiol. Aging 68, 185-198. doi: 10.1016/j.neurobiolaging.2018.05.019

Symmonds, M., Wright, N. D., Bach, D. R., and Dolan, R. J. (2011). Deconstructing risk: separable encoding of variance and skewness in the brain. Neuroimage 58, 1139-1149. doi: 10.1016/j.neuroimage.2011.06.087
Tangney, J. P., Baumeister, R. F., and Boone, A. L. (2004). High self-control predicts good adjustment, less pathology, better grades, and interpersonal success. J. Pers. 72, 271-324. doi: 10.1111/j.0022-3506.2004.00263.x

Tobler, P. N., Christopoulos, G. I., O’Doherty, J. P., Dolan, R. J., and Schultz, W. (2009). Risk-dependent reward value signal in human prefrontal cortex. Proc. Natl. Acad. Sci. 106, 7185-7190. doi: 10.1073/pnas.0809599106

Vohs, K. D., and Faber, R. J. (2007). Spent resources: self-regulatory resource availability affects impulse buying. J. Consum. Res. 33, 537-547. doi: 10.1086/ 510228

Volkow, N. D., and Fowler, J. S. (2000). Addiction, a disease of compulsion and drive: involvement of the orbitofrontal cortex. Cerebral Cortex 10, 318-325. doi: $10.1093 /$ cercor/10.3.318

Volz, K. G., Schubotz, R. I., and von Cramon, D. Y. (2003). Predicting events of varying probability: uncertainty investigated by fMRI. Neuroimage 19, 271-280. doi: 10.1016/S1053-8119(03)00122-8

Volz, K. G., Schubotz, R. I., and von Cramon, D. Y. (2005). Variants of uncertainty in decision-making and their neural correlates. Brain Res. Bull. 67, 403-412. doi: 10.1016/j.brainresbull.2005.06.011

Wang, Y., Liu, Y., Yang, L., Gu, F., Li, X., Zha, R., et al. (2015). Novelty seeking is related to individual risk preference and brain activation associated with risk prediction during decision making. Sci. Rep. 5:10534. doi: 10.1038/srep 10534

Wen, F., He, Z., Gong, X., and Liu, A. (2014). Investors' risk preference characteristics based on different reference point. Discrete Dynam. Nat. Soc. 2014:158386. doi: 10.1155/2014/158386

Wulfert, E., Safren, S. A., Brown, I., and Wan, C. K. (1999). Cognitive, behavioral, and personality correlates of hiv-positive persons' unsafe sexual behavior. J. Appl. Soc. Psychol. 29, 223-244. doi: 10.1111/j.1559-1816.1999.tb0 1383. $x$

Yan, C., and Zang, Y. (2010). DPARSF: a MATLAB toolbox for" pipeline" data analysis of resting-state fMRI. Front. Syst. Neurosci. 4:13. doi: 10.3389/fnsys. 2010.00013

Yan, C.-G., Cheung, B., Kelly, C., Colcombe, S., Craddock, R. C., Di Martino, A., et al. (2013). A comprehensive assessment of regional variation in the impact of head micromovements on functional connectomics. Neuroimage 76, 183-201. doi: 10.1016/j.neuroimage.2013.03.004

Conflict of Interest Statement: The authors declare that the research was conducted in the absence of any commercial or financial relationships that could be construed as a potential conflict of interest.

Copyright (c) 2019 Wang, Chen, Zhang, Xu, Zhang, Suo and Feng. This is an openaccess article distributed under the terms of the Creative Commons Attribution License (CC BY). The use, distribution or reproduction in other forums is permitted, provided the original author(s) and the copyright owner(s) are credited and that the original publication in this journal is cited, in accordance with accepted academic practice. No use, distribution or reproduction is permitted which does not comply with these terms. 